\title{
GECN: Primary Voltage Control for Active Distribution Networks via Real-Time Demand-Response
}

\author{
Konstantina Christakou, Member, IEEE, Dan-Cristian Tomozei, Member, IEEE, Jean-Yves Le Boudec, Fellow, \\ IEEE, Mario Paolone, Senior Member, IEEE
}

\begin{abstract}
Demand Response (DR) has traditionally targeted peak shaving for the optimal allocation of the electricity consumption on a time scale that ranges from minutes to hours. However, with the availability of advanced monitoring and communication infrastructure, the potential of real-time DR for providing ancillary services to the grid has not yet been adequately explored. In this work, we propose a low-overhead decentralized DR control mechanism, henceforth called Grid Explicit Congestion Notification (GECN), intended for deployment by Distribution Network Operators (DNOs) to provide ancillary services to the grid by a seamless control of a large population of elastic appliances. Contrary to classic DR approaches, the proposed scheme aims to continuously support the grid needs in terms of voltage control by broadcasting low-bit rate control signals on a fast time scale (i.e., every few seconds). Overall, the proposed DR mechanism is designed to (i) indirectly reveal storage capabilities of end-customers and (ii) have a negligible impact on the end-customer. In order to estimate the benefits of the proposed mechanism, the evaluation of the algorithm is carried out by using the IEEE 13 nodes test feeder in combination with realistic load profiles mixed with non-controllable demand and non-dispatchable generation from photovoltaic distributed generation.
\end{abstract}

Index Terms-Real-time Demand Response, ancillary services, voltage control, elastic demand, probabilistic load control.

\section{INTRODUCTION}

D emand Response (DR) includes all intentional modifications to the consumption patterns of end-use electrical grid customers, which result in altering the time, the level of instantaneous demand, or the total electricity consumption. The majority of existing DR schemes target peak shaving and, in general, alter the total electricity consumption on a time scale of minutes up to several hours (e.g., [1]). However, with the increasing availability of advanced monitoring and communication technologies, it is also possible to envision using real-time DR mechanisms in order to engage large populations of small electrical loads to provide grid ancillary services (e.g., [2]).

In this direction, in [3] DR is deployed to mitigate forecast errors due to the integration of renewable resources, whereas in [4] DR is considered in the context of islanded microgrids where it aims at providing a form of reserve. Furthermore, inspired by traditional frequency droop controls, there has

Konstantina Christakou, Dan-Cristian Tomozei, Jean-Yves Le Boudec and Mario Paolone (email: konstantina.christakou@epfl.ch, dan-cristian.tomozei @epfl.ch, jean-yves.leboudec@epfl.ch, mario.paolone@epfl.ch) are with the École Polytechnique Fédérale de Lausanne, CH-1015 Lausanne, Switzerland. already been an effort to investigate DR schemes as a way to provide primary and secondary frequency control to the grid. In particular, in [5] electric vehicles are considered for providing frequency control, whereas in [6] domestic loads are investigated for primary frequency control. In this respect, it is worth noting that this type of DR contribution to frequency control appears interesting in the case of islanded grids but, as it was recently requested by the European Network of Transmission System Operators for Electricity (ENTSO-E), it might be extended to distribution networks that will be requested to provide grid ancillary services (e.g., [7]).

The purpose of this work is to develop a new DR control mechanism for providing a different ancillary service, specifically the primary voltage control of active distribution networks. This specific control is becoming increasingly compelling due to the progressive penetration of distributed energy resources in this specific layer of the electrical infrastructure. Within the context of voltage control of distribution networks, it is important to underline that this specific ancillary service requires controlling both active and reactive power injections, in view of the non-negligible $R / X$ ratio of longitudinal parameters of the medium and low voltage lines (e.g., [8], [9] $)^{1}$.

In order to investigate the potential of a large aggregation of small electrical loads for providing primary voltage control, this paper proposes the use of a low-overhead decentralized DR control scheme inspired by the congestion control mechanism used in the Transmission Control Protocol (TCP) [10], henceforth called the Grid Explicit Congestion Notification (GECN). Specifically, the proposed mechanism is designed to provide ancillary services to the grid by means of a low bit-rate control signal, broadcasted to a large population of controlled appliances. These appliances, in our case, consist of thermostatic loads (e.g., space/water heating, refrigeration). These kinds of loads are a promising category for engaging in short-term ancillary services as they are typically characterized by slow-evolving states (e.g., temperature with hourly time dynamics) that allow for control flexibility (e.g., [11], [12], [13]).

Contrary to classic DR approaches, GECN acts on a fast time scale (in the order of few seconds) without significantly impacting the end customers. Within the context of distribu-

\footnotetext{
${ }^{1}$ The control of the active power can be rewarded by the DNO as it is "de-facto" an ancillary service for which an unbundled market might provide specific contracts.
} 
tion networks voltage control, the major contributions of the proposed mechanism are as follows:

- Under normal grid operation, the proposed scheme can be used similarly to classic DR schemes for peak shaving or for continuously maintaining the balance between generation and consumption in the network (when there is not enough capacity or when there are renewable resources whose generation cannot be fully predicted). Furthermore, it is easily adapted to balance load/generation in cases of intentional islanding;

- In emergency situations, such as contingencies in the main grid, the proposed scheme can be used to provide a further control leverage on the reactive power requested by distribution networks;

- Finally, the proposed mechanism takes into account the issue of limiting the frequency of cycles of the elastic appliances and avoids possible synchronization (i.e., cold load pick up [14]) after the DR actions.

The structure of the paper is the following: in Section II the representation of both elastic and non-elastic appliances is described. Section III, focuses on the detailed description of GECN; both the elastic load response, and the network operator's required actions are presented. In Section IV the evaluation of the proposed scheme is provided through application examples using the IEEE 13 nodes test feeder. Finally, Section V provides the final remarks about possible future applications of the proposed mechanism.

\section{LOAD RESPONSE REPRESENTATION}

In this section, we give the main assumptions on both elastic and non-elastic demand. Let us consider thermostatic loads for which their state is given by their temperature (e.g., air conditioners or refrigerators). We consider $L$ types of elastic appliances. We assume that each type $\ell$ has a set of operating modes that we denote by $\mathcal{X}_{\ell} \subseteq[0,1]$, a maximum rated power $P_{\ell}^{r}$, and a constant power factor $\cos \varphi_{\ell}$. When an appliance of type $\ell$ is in a specific operating mode $x \in \mathcal{X}_{\ell}$, it consumes a fraction $x$ of the rated power, i.e., $x P_{\ell}^{r}$ and the corresponding proportional reactive power, obtained via the power factor.

In what follows, we consider discrete time steps $\{0,1, \ldots, t, \ldots\}$. Let us consider a single appliance of type $\ell$. We denote the process describing its operating mode by $\left(X(t) \in \mathcal{X}_{\ell}\right)_{t}$. The internal state process of the appliance is influenced by the operating mode and by external factors; we denote it by $(\theta(t) \in \mathbb{R})_{t}$. At all times $t \geq 0$, the controller of the appliance chooses an operating mode $X(t)$ such that the internal state constraints $\Theta_{\ell} \subset \mathbb{R}$ are satisfied, i.e., $\theta(t) \in \Theta_{\ell}$.

The duty-cycle function $h_{\ell}: \mathcal{X}_{\ell} \times \mathbb{R} \rightarrow \mathcal{X}_{\ell}$ for an appliance of type $\ell$ determines the default operating mode of the appliance $X(t+1)$, taking into account the previous operating mode $X(t)$ and the internal state of the appliance $\theta(t)$ :

$$
X(t+1)=h_{\ell}(X(t), \theta(t)), t \geq 0 .
$$

For presentation ease, we describe the behavior of a cooling thermostatic device. Such an appliance is assumed to operate in a binary mode $\mathcal{X}=\{0,1\}$, and its internal state is given by the cooling compartment temperature, constrained in the form of a deadband $\Theta=\left[\theta_{\min }, \theta_{\max }\right]$ (e.g., [15]). The internal temperature of these kinds of loads can be modeled, for instance, as proposed in [16]:

$$
\theta(t+1)=\epsilon \theta(t)+(1-\epsilon)\left(\theta_{0}-\eta \frac{X(t) P_{r}}{A}\right)+\omega(t)
$$

where $\theta_{0}$ is the ambient temperature, $\epsilon=e^{-\tau A / m_{c}}$ is the factor of inertia of the appliance, $\tau$ is the time step and $m_{c}$ is the thermal mass, $\eta$ is its coefficient of performance, and $A$ is the thermal conductivity. The process $\omega(t)$ is a noise process-modeling of the random external heat injections in thermostatically controlled loads having a distribution that follows hourly data presented in [3]. A typical duty-cycle function (Fig. 1) is:

$$
\begin{aligned}
& h(X=0, \theta)=\mathbb{1}_{\left\{\theta \geq \theta_{\max }\right\}} \\
& h(X=1, \theta)=\mathbb{1}_{\left\{\theta \geq \theta_{\min }\right\}}
\end{aligned}
$$

In the rest of the paper, we consider a distribution network comprising $M$ 3-phase buses and at each bus $i$ a population of the aforementioned elastic appliances, each one having a state that evolves like Eq. 2 and a duty cycle function given by Eq. 3. In addition to the controllable loads, in each network bus, non-elastic demand, non-controllable by the DNO is assumed, represented by typical $24-\mathrm{hr}$ load profiles. The aggregate power at a bus level consists of the combination of the above types of loads.

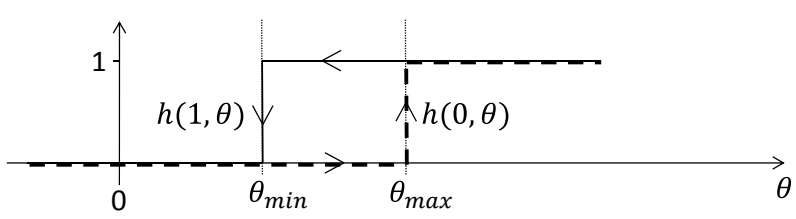

Fig. 1. Duty-cycle for appliances with deadband-constrained state.

\section{GECN:DEMAnd RESPONSE CONTROL MECHANISM}

In this section, we describe the basic principles and operations of GECN, a load-control mechanism that provides "distributed" ancillary services to the grid via a seamless control of a large population of elastic appliances. First, the DNO estimates the aggregate power at all the network buses. At each bus both elastic and non-elastic loads are present, as well as non-dispatchable active power injections provided by distributed generators. The DNO does not aim to individually control each appliance. Instead, it broadcasts on each bus a unique control signal that is transparent to the non-elastic appliances. Therefore, this signal impacts solely the behavior of the elastic loads. The bit rate of the signal is very low (a few bits per second), therefore it can be transmitted using existing power line communication for advanced metering ${ }^{2}$. Note that we cannot use frequency deviation as alternative to an explicit broadcast control signal, since we are targeting voltage control and voltage deviations in a local bus may appear independently of frequency deviations.

\footnotetext{
${ }^{2}$ It is worth mentioning that details of the telecommunication infrastructure are outside the scope of this paper.
} 
Each elastic appliance is assumed to be equipped with a simple programmable controller that decides its power consumption based on the internal state and the received signal. The resulting variation of the aggregate power at the bus provides the DNO with an implicit feedback to the control signal, which is used to estimate the responsiveness of the bus loads and to decide the subsequent control signal.

In what follows we describe in detail the operations of the controller of the elastic appliances as well as the DNO's actions that determine the optimal GECN control signal.

\section{A. Load Controller}

All the elastic appliances connected to a network bus $i$ receive at each time step $t$ the GECN control signal $g_{i}(t)$ broadcasted by the DNO. The signal represents a real number $g_{i}(t) \in[-1,1]$ coded, for example, on 16 bits. The control signal $g_{i}(t)$ is proportional to the DNO's desire to inhibit consumption. Hence, a negative $g_{i}$ encourages consumption, a positive $g_{i}$ inhibits consumption, whereas $g_{i}=0$ does not impact the behavior of the appliance ${ }^{3}$.

In what follows, we propose a controller that takes into account the GECN signal. In the absence of the GECN control signal (i.e., $g_{i}(t)=0$ ), the duty-cycle function in Eq. 3 provides the controller with the next operating mode $X(t+1)$. In response to a non-zero GECN signal, the controller can decide to switch to a different operating mode than the one dictated by the duty-cycle. When such a decision is made, the controller sets a counter $T(t)$ to a predefined value $T_{\ell, 0}$ (which depends on the appliance type); the value $T(t)$ then decreases over time, until it reaches 0 . When the value of $T(t)$ is strictly positive, the appliance does not react to any GECN signals. This ensures a smooth operation of the appliance by limiting the number of imposed operating mode switches.

For presentation ease, in what follows we define the operation of the controller for a cooling thermostatic device as described in Sec. II. For such an appliance, at each time step, the controller decides the next value $X(t+1)$ as a function of $X(t)$, its current state $\theta(t)$ (temperature), and the GECN signal $g_{i}(t)$ as follows:

1) If the value of the counter $T(t)$ is not equal to zero, then the appliance disregards the GECN signal and continues normal operation, as previously explained.

2) If $T(t)=0$, the intensity of the signal is taken into account: upon receiving $g_{i}(t)$ the controller draws a Bernoulli random variable $Y$ with parameter $p=\left|g_{i}(t)\right|$ (i.e., $Y=1$ with probability $p$ and $Y=0$ with probability $1-p$ ) that reflects the desired response by the DNO. If $Y$ is set to 1 , then the appliance proceeds to the next step, otherwise it continues with normal operation.

3) Finally, if $Y=1$, the actual response of the appliance depends on its current operating mode $X(t)$ and internal

\footnotetext{
${ }^{3}$ The proposed algorithm aims at maximizing the usage of network resources (i.e., loads) to locally provide ancillary services to the grid. In this respect, an imbalance of the production/consumption towards the production encourages consumption, whereas an imbalance towards the consumption inhibits consumption in order to keep the voltage levels within the allowed limits.
}

state $\theta(t)$. Specifically, the controller draws a second, independent Bernoulli random variable $Z$ with parameter $q\left(X(t), \theta(t), g_{i}(t)\right)$, where $q(x, \vartheta, g)$ characterizes the appliance's propension to switch operating mode given that $X(t)=x, \theta(t)=\vartheta$, and $g_{i}(t)=g$. The appliance then switches state if $Z=1$, else it does not.

For example, when the cooling appliance is on, the closer the temperature is to the maximum $\theta_{\max }$, the less likely it is that the appliance responds to a signal requiring it to switch off. Thus, when the DNO wants to inhibit consumption $(g>0)$ and the appliance is on, the probability $q$ should be chosen so that $q=1$ whenever $\vartheta<\theta_{\min }, q=0$ whenever $\vartheta>\theta_{\max }$, and decreasing with $\vartheta$ in $\vartheta \in\left[\theta_{\min }, \theta_{\max }\right]$.

We use $q(x, \vartheta, g)=\min \{\tilde{q}(x, \vartheta, g), 1\}$ where

$\tilde{q}(x, \vartheta, g)= \begin{cases}C x\left[1-e^{-\left(\theta_{\max }-\vartheta\right) / \xi}\right]^{+} & \text {if } g \geq 0 \\ C(1-x)\left[1-e^{-\left(\vartheta-\theta_{\min }\right) / \xi}\right] & \text { if } g<0\end{cases}$

and $C=\left[1-e^{-\left(\theta_{\max }-\theta_{\min }\right) / \xi}\right]^{-1}$ is a normalization constant ${ }^{4}$.

We can summarize the proposed controller's response to the GECN signal as

$$
X(t+1)= \begin{cases}1-X(t), & \text { if } Y=Z=1, \\ h(X(t), \theta(t)), & \text { otherwise. }\end{cases}
$$

Thus an appliance can disregard a GECN $g_{i}(t)$ signal for three reasons: (i) a too recent reaction to the GECN signal, in other words, the counter $T(t)$ is not equal to 0 - this avoids the appliance operation in "mini-cycles", (ii) a small magnitude of $g_{i}(t)$, implying low requirement from the DNO side, or (iii) an inopportune internal state of the appliance.

\section{B. GECN Integration Into Primary Voltage Control}

As anticipated, the DNO wishes to use GECN signals to perform primary voltage control. In this section we describe a method to compute such signals.

The DNO is assumed to have imperfect 24-hr forecasts for load and renewable profiles $(\mathbf{P}, \mathbf{Q})_{f}$, from which deviation is penalized in order to reduce costs of operation. Finally, the operator is assumed to be able to control the primary substation On-Load Tap-Changers (OLTC) located in correspondence to the slack bus of the network ${ }^{5}$. The tap-changers positions are a means to provide a further leverage on the operator in coordination with the proposed control mechanism.

At each time-step $t$ the DNO observes the state of the network on every bus $i$, i.e., the per-bus aggregate power injections $P_{i}(t)$ and $Q_{i}(t)$, along with the phasors of the phase to ground voltage $\bar{E}_{i}(t)$. This information is assumed to be obtained via a state estimation algorithm (e.g., [17]). Subsequently, the DNO computes the voltage sensitivity coefficients

\footnotetext{
${ }^{4}$ We used the notation $[a]^{+}:=\max (a, 0)$. The denominator of the exponent, $\xi$, has been selected empirically to modulate the appliances' response to the GECN signal. However, such a coefficient does not influence the behavior of the proposed control mechanism.

${ }^{5} \mathrm{We}$ disregard the presence of var compensators. Note that they can be taken into account in the $\mathbf{K}_{\mathbf{Q}}$ matrix.
} 
with respect to absorbed/injected power of a bus $\ell$, as well as transformer's tap-changers positions

$$
\begin{gathered}
K_{P, i \ell}(t):=\frac{\partial\left|\bar{E}_{i}\right|}{\partial P_{\ell}}(t), K_{Q, i \ell}(t):=\frac{\partial\left|\bar{E}_{i}\right|}{\partial Q_{\ell}}(t) \\
K_{n, i}(t):=\frac{\partial\left|\bar{E}_{i}\right|}{\partial E_{\text {slack }}}(t)
\end{gathered}
$$

e.g., by solving the linear systems of equations presented in [8], [18]. Therefore, the following linear relation between variation in bus voltages and variations of active/reactive power $\Delta P_{i}, \Delta Q_{i}$ and tap-changers positions $\Delta n$ can be derived (e.g. [19]):

$$
\begin{aligned}
\Delta|\bar{E}|_{i} & \approx \mathbf{K}_{\mathbf{P} i} \boldsymbol{\Delta} \mathbf{P}+\mathbf{K}_{\mathbf{Q}_{i}} \boldsymbol{\Delta} \mathbf{Q}+\mathbf{K}_{\mathbf{n}} \mathbf{\Delta} \mathbf{n} \\
& \triangleq\left(\mathbf{K}_{\mathbf{P}, \mathbf{Q}, \mathbf{n}}(t) \boldsymbol{\Delta}(\mathbf{P}, \mathbf{Q}, \mathbf{n})\right)_{i} .
\end{aligned}
$$

where by using the same nomenclature of [8] $\mathbf{K}_{\mathbf{P} i}=\left[K_{P_{i 1}}, \ldots, K_{P_{i M}}\right], \quad \mathbf{K}_{\mathbf{Q}_{i}}=\left[K_{Q_{i 1}}, \ldots, K_{Q_{i M}}\right] \quad$ and $\mathbf{K}_{\mathbf{n}}=\left[K_{n_{1}}, \ldots, K_{n_{M}}\right]$.

At this stage the DNO can detect which areas of the network are congested or exhibiting voltage variations close to the limits allowed for safe operation (typically $\pm 5 \%$ of the rated value). Using the sensitivity coefficients $\mathbf{K}_{\mathbf{P}, \mathbf{Q}, \mathbf{n}}$ the DNO can compute the optimal required power adjustments $\left\{\boldsymbol{\Delta}(\mathbf{P}, \mathbf{Q})^{*}(t)\right\}$ in the buses and, at the same time, the optimal variations in the tap-changers positions $\left\{\boldsymbol{\Delta} \mathbf{n}^{*}(t)\right\}$ which lead to the desired operation set-point for voltage control via the following constrained optimization problem:

$$
\begin{gathered}
\min _{\boldsymbol{\Delta}(\mathbf{P}, \mathbf{Q}, \mathbf{n})} \sum_{i} \mu_{i}\left(\boldsymbol{\Delta}(\mathbf{P}, \mathbf{Q})_{i}-\boldsymbol{\Delta}(\mathbf{P}, \mathbf{Q})_{f_{i}}\right)^{2}+ \\
\sum_{i} \lambda_{i}\left[\left(\left|\bar{E}_{i}\right|+\left(\mathbf{K}_{\mathbf{P}, \mathbf{Q}, \mathbf{n}}(t) \boldsymbol{\Delta}(\mathbf{P}, \mathbf{Q}, \mathbf{n})\right)_{i}-|\bar{E}|\right)^{2}-\alpha^{2}\right]^{+}+ \\
\psi_{1}\left(\sum_{i} \hat{g}_{i}\right) \psi_{2}\left(\sum_{t}|\Delta n(t)|\right) \Delta \mathbf{n}^{2} \\
\text { subject to: } \quad \gamma_{i} \leq \cos \varphi_{i} \leq 1 \\
n_{\min } \leq n(t) \leq n_{\max } \\
\text { where } \quad \hat{g}_{i}(t)=\sum_{s=0}^{W-1} k_{s} g_{i}(t-s) \\
\text { and } \quad n(t)=n\left(t_{0}\right)+\sum_{\tau=t_{0}}^{t} \Delta n(\tau)
\end{gathered}
$$

$\gamma_{i}$ is the constraint on the power factor, $\cos \varphi_{i}$, of a specific bus $i, n_{\min }$ and $n_{\max }$ are the minimum and maximum OLTC positions allowed, the parameter $\alpha$ denotes the value of the voltage deviation from the rated value tolerated by the DNO, $\hat{g}_{i}$ is the moving average of the control signal $g$ over a time window of $W$ time steps, and $\psi_{1}, \psi_{2}$ are penalty functions for altering the tap-changer position ${ }^{6}$. The first two terms in the objective function are weighted by parameters $\lambda_{i}$ and $\mu_{i}{ }^{7}$. The first term of Eq. 5 represents the compensation of errors

\footnotetext{
${ }^{6}$ As we deal with primary voltage control, Eq. 5 has to penalize the changes of OLTC as these devices are typically used by the DNO rarely. This will be explained next with more details.

${ }^{7}$ The choice of the weights in the objective function is related to the topology of the network and the parameters of the lines (i.e., the network admittance matrix).
}

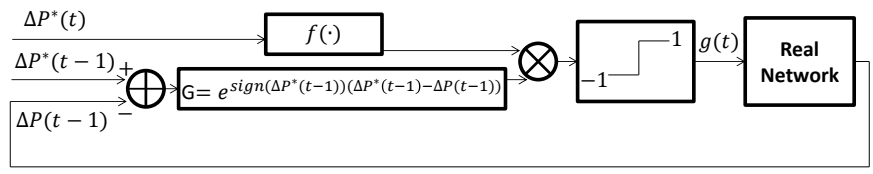

Fig. 2. Control loop for GECN signal $\mathbf{g}(t)$.

in the forecasted aggregated loads. The second and third terms describe the voltage control part. The operator can perform this type of control by deploying solely demand response or by coordinating control of the loads and the OLTC positions. In the case where the tap-changers are included, the DNO seeks to utilize them only in periods when demand response cannot provide the desirable operating set points. This is represented by the term $\psi_{1}$ of Eq. 5 which is a function that assumes large values in normal operation (i.e., $|\hat{g}| \simeq 0$ ) and low values when the GECN signal saturates (i.e., $|\hat{g}| \simeq 1$ ). More specifically, we have chosen:

$$
\psi_{1}\left(\sum_{i} \hat{g}_{i}\right):=a_{1} e^{-a_{2}\left|\sum_{i} \hat{g}_{i}\right|}
$$

In order to account for the limited number of operations that an OLTC can perform, a further penalty function, $\psi_{2}$, has been included in Eq. 5. This function multiplies the OLTC set-points variation and increases with the number of OLTC operations in a given time window ${ }^{8}$. Specifically, we have chosen:

$$
\psi_{2}\left(\sum_{t}|\Delta n(t)|\right):=e^{a_{3} \sum_{t_{i}<t<t_{i}+\tau_{o}} a_{4}\left(n\left(t_{i}\right) \Delta n\left(t_{i}\right)\right)\left|\Delta n\left(t_{i}\right)\right|}
$$

where $a_{3}$ is a constant and $a_{4}$ is 1 if $n(t) \Delta n(t) \geq 0$ and 0 otherwise. Such an expression of $\psi_{2}$ allows to weight in an exponential way the accumulated number of OLTC changes within a given time window $\tau_{o}$. Furthermore, it is able to account for the direction of the OLTC changes together with their distance from the central OLTC position $n=0$. In view of the expression used to weight the penalty on $\Delta n$, the DNO can decide the leverage on the OLTC compared to the use of other resources (i.e., storage and/or loads).

After having determined the optimal operation set-points of the network at time $t$, the goal is to adaptively compute GECN signals which drive the system towards these desired set-points. This is implemented using the control loop depicted in Fig. 2. The GECN signal at time $t$ is computed as a function of (i) the optimal set points at the current time step and (ii) the mismatch between the optimal and the actual set points that the DNO observed at the previous time step $t-1$. The current optimal set points are the input to a saturation function $f$ that maps $\Delta P_{i}^{*}(t)$ every time-step to a value in $[-1,1]$ :

$$
f\left(\Delta P_{i}^{*}(t)\right)=\operatorname{sign}\left(\Delta P_{i}^{*}(t)\right)\left(1-e^{-\left|\Delta P_{i}^{*}(t)\right| / b_{i}}\right)
$$

where $\Delta P_{i}^{*}(t)$ are the optimal set points that the DNO targets at time $t$, and $b_{i}$ is a parameter affecting the slope of the

\footnotetext{
${ }^{8}$ By including this function, even in cases where the saturation of the signal occurs for a long period of time, the DNO has the option to upper-bound the total number of OLTC operations, thus respecting the nature and cost of these devices.
} 
saturation function (i.e., the smallest the value of $b$, the steepest the slope of the saturation function). The outcome of this operation is weighted by a factor $G$, which is an exponential function of the mismatch between the optimal set points and the actual set points the DNO observes at the previous time step $t-1$ :

$$
G=e^{\operatorname{sign}\left(\Delta P_{i}^{*}(t-1)\right)\left(\Delta P_{i}^{*}(t-1)-\Delta P_{i}(t-1)\right)}
$$

Thus, the proposed control loop continuously adapts the GECN signal to adjust the aggregate power at each bus to the variations of the optimal set points.

\section{Solver and Computational Performance}

In Eq. 5 the variation of the tap-changers position $\Delta n$ is a discrete variable. Therefore, when the DNO wishes to include the variation of the tap-changers, the problem becomes a mixed integer non linear programming (MINLP) one. In order to solve the optimization problem a MATLAB/GAMS interface is used [20]. In GAMS the KNITRO solver [21] is used to find the solution to both the MINLP, when the taps are included and the NLP, when the DNO disregards the presence of the tap-changers.

For the considered 24-hr horizon the optimization problem is solved every 16 seconds resulting in 5400 execution times. Table I shows the mean CPU times necessary to solve the MINLP, as well as the NLP, with reference to an Intel CORE i7, $2.80 \mathrm{GHz}$, 4GB RAM, running Windows 7. In the same table the relevant 95 -th percentiles are also reported. It can be observed that the time required to solve the optimal control problem with a standard laptop is compatible with a real-time use of the proposed approach. Indeed, as shown in Table I, for the selected time step ( 16 seconds) the optimal problem is solved in the order of some hundreds of ms.

Table I

CPU TIME NECESSARY FOR SOLVING THE OPTIMIZATION PROBLEM IN EQ. 5

\begin{tabular}{|c|c|c|}
\cline { 2 - 3 } \multicolumn{1}{c|}{} & Mean $(\mathrm{ms})$ & 95-th Percentile $(\mathrm{ms})$ \\
\hline MINLP & 181.2 & 194.7 \\
\hline NLP & 168.2 & 186.8 \\
\hline
\end{tabular}

\section{ApPLICATION OF GECN}

For the evaluation of the proposed algorithm we have considered a modified IEEE 13 nodes test feeder as depicted in Fig. 3. The modifications are: (i) balanced lines, (ii) inclusion of secondary substations (henceforth called "aggregators") where voltage-independent PQ equivalents are placed and, (iii) lines ten times longer. As a consequence, using the approach described in [8], we can compute the network voltage sensitivity coefficients. A specific Matlab code has been developed to solve the network load flow problem and to implement the GECN control mechanism.

Each aggregator in the network comprises a large population of heterogeneous household controllable loads along with nonelastic demand, as well as non-dispatchable power injections. The technical characteristics of the elastic loads and the load controller parameters are given in Table II ([22], [3],

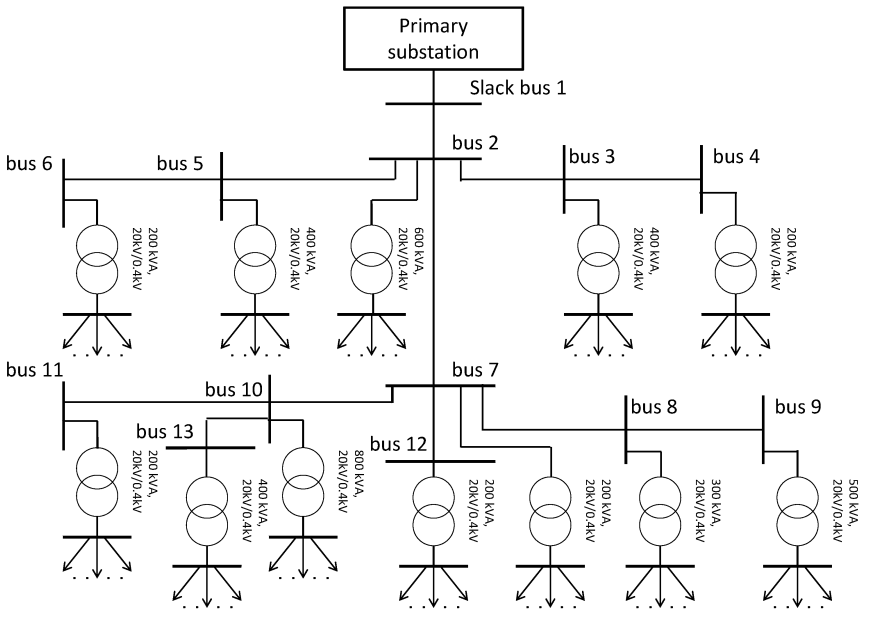

Fig. 3. Modified IEEE 13 nodes test feeder used for the evaluation of the proposed DR mechanism.

Table II

PARAMETERS OF THE ELASTIC APPLIANCES AND THE LOAD CONTROLLER

\begin{tabular}{|c|c|}
\hline Parameter & Value \\
\hline Temperature deadband, $\Theta\left({ }^{\circ} \mathrm{C}\right)$ & {$[3,8]$} \\
\hline Ambient temperature, $\theta_{0}\left({ }^{\circ} \mathrm{C}\right)$ & $\sim U(10,15)$ \\
\hline Thermal conductivity, $\mathrm{A}\left(\mathrm{kW} /{ }^{\circ} \mathrm{C}\right)$ & 10.563 \\
\hline Coefficient of performance, $(\eta)$ & 1.5 \\
\hline Rated power, $P_{r}($ Watt $)$ & 150 \\
\hline Time step, $\tau(\mathrm{sec})$ & 1 \\
\hline Time constant, $T_{c}=m_{c} / A(\mathrm{hrs})$ & $\sim U(1.326,2.778)$ \\
\hline Controller time counter, $T_{0}(\mathrm{sec})$ & 480 \\
\hline Internal state parameter, $\xi$ & 0.4 \\
\hline Appliance power factor, $\cos \varphi$ & 0.85 \\
\hline Parameters of the penalty function $\psi_{1}, a_{1}, a_{2}$ & 1000,2 \\
\hline Parameters of the penalty function $\psi_{2}, a_{3}, \tau_{o}(\mathrm{hrs})$ & 5,3 \\
\hline Window of $\hat{g}, W$ (time steps) & 3 \\
\hline
\end{tabular}

[16]). Concerning the non-dispatchable power generation, we assumed to have a typical PV-type profile with peak power that changes for all aggregators within the range of $90 \%-180 \%$ of each secondary substation peak load. Additionally, Fig. 4 illustrates the total daily load and power injections profile in the network, where the convention used is that negative values represent power injection and positive power consumption.

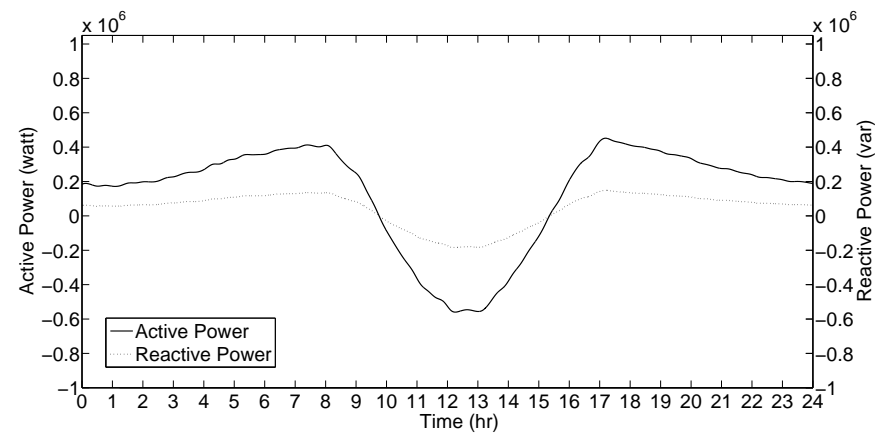

Fig. 4. Total daily load and power injections profile in the network.

We assumed three different test cases for the available elastic demand in the network, $10 \%, 20 \%$ and $30 \%$ of the total peak load. In Table III, the number of appliances per 
Table III

NUMBER OF ELASTIC APPLIANCES PER NETWORK BUS

\begin{tabular}{|c|c|c|c|}
\hline Bus & $10 \%$ elastic demand & $20 \%$ elastic demand & $30 \%$ elastic demand \\
\hline 1 & - & - & - \\
\hline 2 & 93 & 182 & 310 \\
\hline 3 & 113 & 204 & 328 \\
\hline 4 & 112 & 192 & 309 \\
\hline 5 & 114 & 216 & 324 \\
\hline 6 & 100 & 210 & 304 \\
\hline 7 & 105 & 197 & 336 \\
\hline 8 & 118 & 219 & 320 \\
\hline 9 & 98 & 210 & 325 \\
\hline 10 & 82 & 217 & 327 \\
\hline 11 & 115 & 212 & 311 \\
\hline 12 & 105 & 209 & 314 \\
\hline 13 & 94 & 211 & 305 \\
\hline
\end{tabular}

network bus is shown for these three cases. For each test case two scenarios have been considered. Scenario I relies on the assessment of the voltage controllability by using only the DR control mechanism (by setting the coefficients $K_{n, i}(t)$, and the third term of the objective function in Eq. 5 to zero), whereas Scenario II assumes that the DNO uses the DR control mechanism together with the control of the primary substation OLTC (i.e., with the objective function as described in Eq. 5).

\section{A. Voltage Control}

In this section, the DNO deploys GECN to continuously penalize the deviation of the voltage in the buses of the network in order to guarantee a voltage profile for safe operation. To this end, the operator solves at each time step the optimization problem defined in Eq. 5 with $\lambda_{i}=0.15$ and $\mu_{i}=1$ for both scenarios I and II. Specifically, for scenario II, the moving average of the control signal is computed as $\hat{g}_{i}(t)=0.8 g_{i}(t)+0.1 g_{i}(t-1)+0.1 g_{i}(t-2)$.

The DNO wishes that the voltage deviations in the network busses are in the range of $\pm 5 \%$. In order not to saturate the available DR resources of the network, we assume that there is a tolerance in the voltage deviations from the network-rated value, which is represented by the parameter $\alpha$ in Eq. 5. The role of this parameter is to create a deadband within which the DNO is not interested in minimizing the voltage, thus sacrificing the scarce resources. In this section this parameter is set to $\alpha=0.04$ and the resulting deadband for the voltage profile is $[0.96,1.04] p . u$. Additionally, it is assumed that the constraint $\gamma_{i}$ on the power factor of the aggregate load in all buses is 0.9. Finally, we assume to have an OLTC that can vary the rated value of the network voltage in the range of $\pm 6 \%$, operating in 72 tap positions.

In order to infer the benefits of the proposed mechanism, we assume a base case where the total demand in the network is non-elastic and obtain the daily voltage profile of the network buses. For the sake of brevity, we limit the validation of the proposed method to a reduced number of buses. In particular, we refer mainly to two buses of interest: bus 2 , which is the closest one to the slack bus; and bus 13 , which the furthest one from the slack bus of the network. In Fig. 5 one can observe the voltage profile of these buses for the base case, as well as the improvement in the voltage profile for both

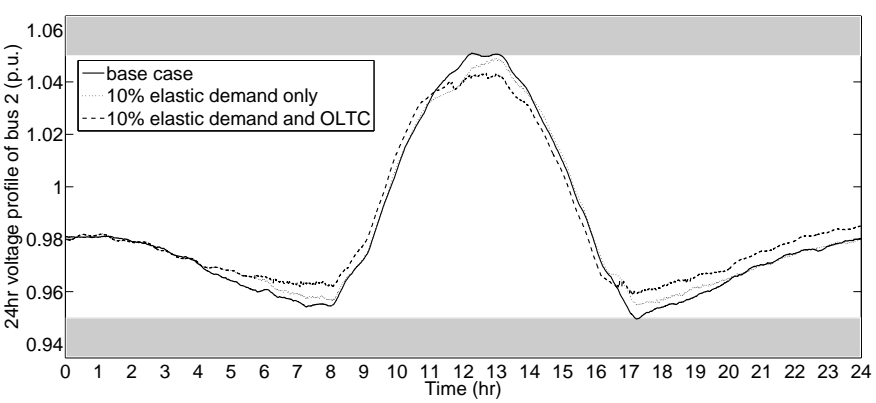

(a) $24 \mathrm{hr}$ voltage profile of bus 2



(b) $24 \mathrm{hr}$ voltage profile of bus 13

Fig. 5. 24hr voltage profiles of bus 2 and 13 for $0 \%$ of elastic demand (base case) and $10 \%$ of elastic demand with (scenario II) and without (scenario I) control of the tap-changers positions.

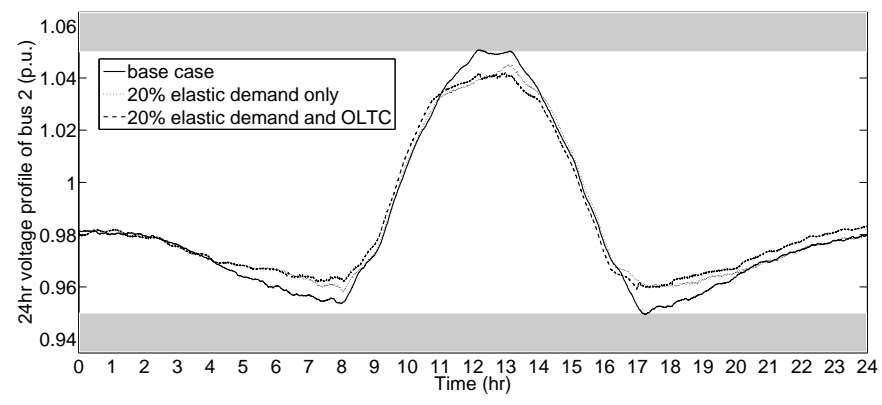

(a) $24 \mathrm{hr}$ voltage profile of bus 2

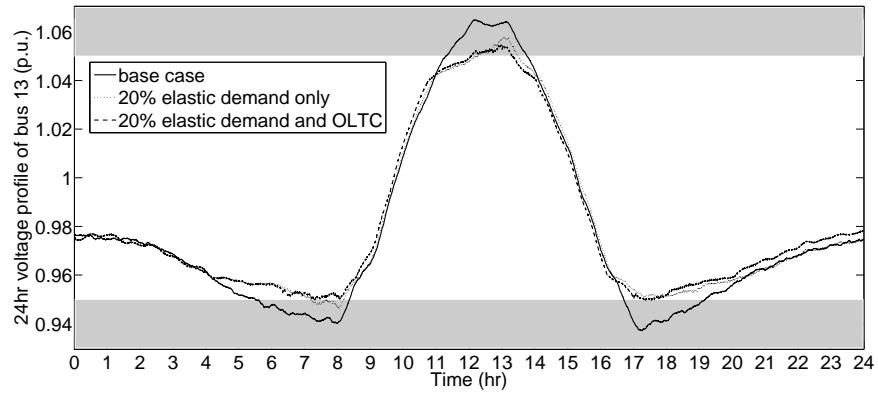

(b) $24 \mathrm{hr}$ voltage profile of bus 13

Fig. 6. $24 \mathrm{hr}$ voltage profiles of bus 2 and 13 for $0 \%$ of elastic demand (base case) and $20 \%$ of elastic demand with (scenario II) and without (scenario I) control of the tap-changers positions.

considered scenarios when there is $10 \%$ of elastic demand in the network. The gray bands indicate the voltage range $[0.95,1.05]$. Fig. 6 and 7 show the same voltage profiles 


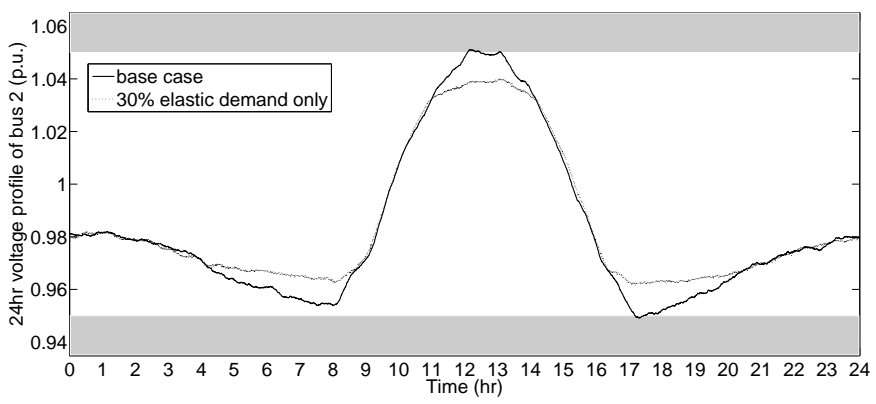

(a) $24 \mathrm{hr}$ voltage profile of bus 2

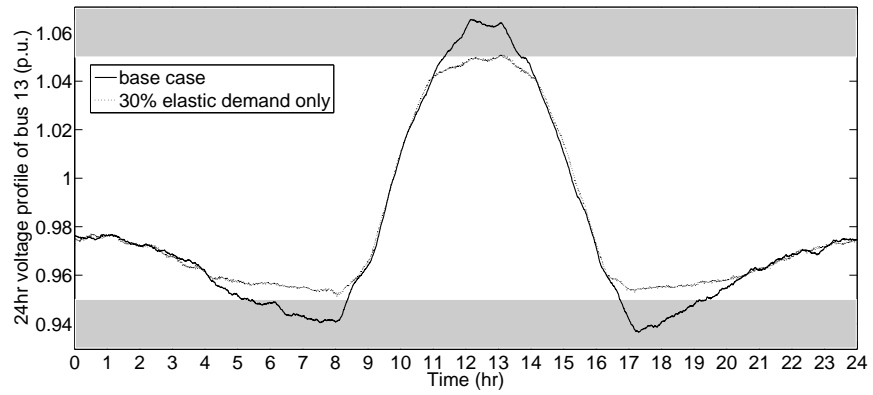

(b) $24 \mathrm{hr}$ voltage profile of bus 13

Fig. 7. 24hr voltage profiles of bus 2 and 13 for $0 \%$ of elastic demand (base case) and $30 \%$ of elastic demand without (scenario I) control of the tap-changers positions.

for the case of $20 \%$ and $30 \%$ elastic demand respectively. Fig. 5 shows an improvement of voltage profiles that can be quantified in the range of $1 \%$ in correspondence of the maximum aggregated power absorption and power production, whereas, Fig. 6 and 7 show an even larger improvement (in the order of $2 \%$ ) due to the increased availability of controllable loads. In fact, for the case of $30 \%$ elastic demand the improvement is only due to the controllable loads as the number of OLTC changes is zero. This is due to the fact that the increased availability of controllable appliances is already enough to guarantee a voltage profile at all buses in the range of $[0.95,1.05]$, thus eliminating the need to leverage on the OLTC. This result reveals the capabilities of the proposed method to enable a non-negligible contribution of controllable loads to voltage control of distribution networks eliminating the need to use other traditional systems like OLTC.

For the same cases as in Fig. 5, 6 and 7, Fig. 8, 9 and 10 show the GECN signals that were sent by the DNO to achieve the desired behavior of the elastic loads for scenarios I and II. It can be observed that the variation of the GECN signals is smoothed by the control leverage of the OLTC. This aspect appears important as it could allow the DNO to decide the better control strategy, namely leveraging more on the load elasticity or on its own resources (i.e., OLTC). These figures also show a large saturation of the GECN signal without the help of the OLTC in the case of $10 \%$ and $20 \%$ of elastic demand. It is also interesting to observe that the increase of elastic loads from $10 \%$ to $20 \%$ directly results in a decrease of the GECN saturation. This allows for an indirect quantification of the DR elasticity and as a consequence an estimation of the

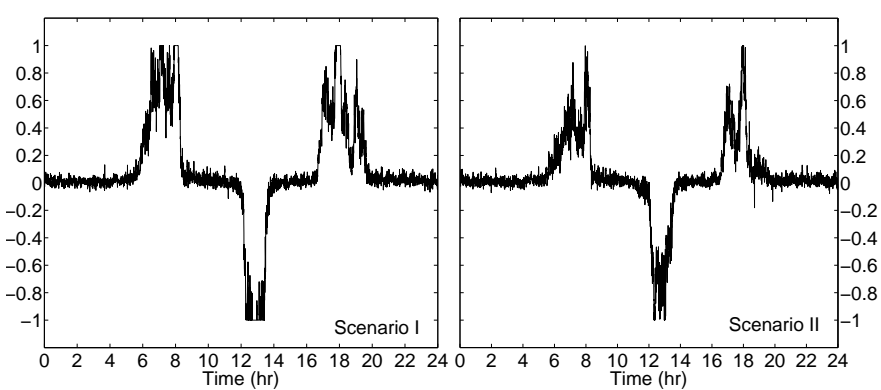

(a) $24 \mathrm{hr}$ GECN signals sent to bus 2

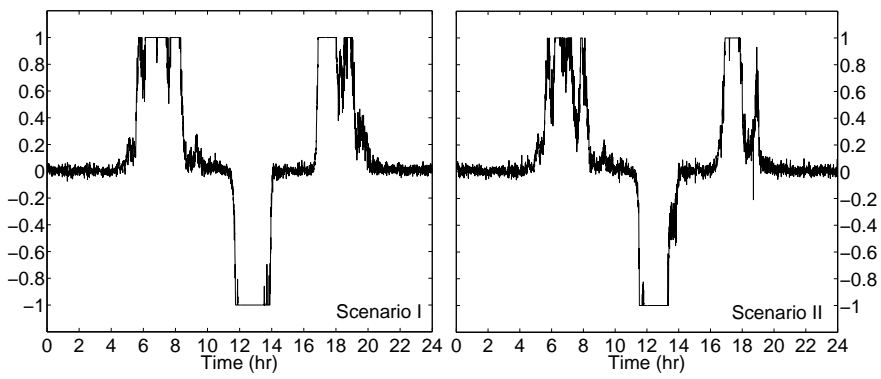

(b) $24 \mathrm{hr}$ GECN signals sent to bus 13

Fig. 8. GECN signal sent to buses 2 and 13 in the case of $10 \%$ of elastic demand with (scenario II) and without (scenario I) control of the tap-changers positions.

optimal number of OLTC changes allowed per day.

Fig. 11 shows the daily profile of the OLTC changes

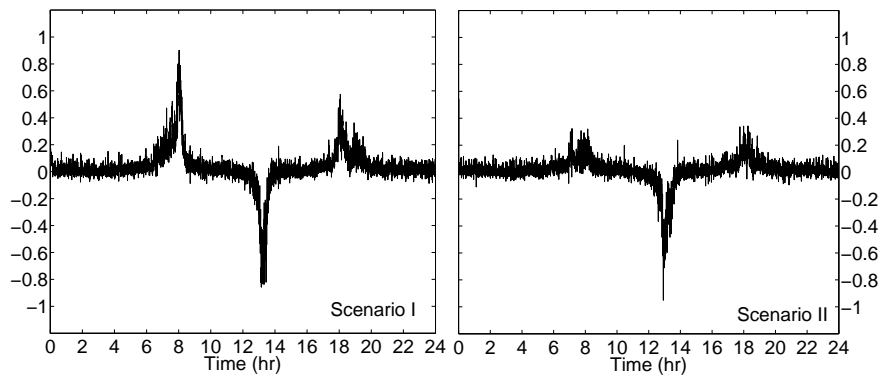

(a) $24 \mathrm{hr}$ GECN signals sent to bus 2

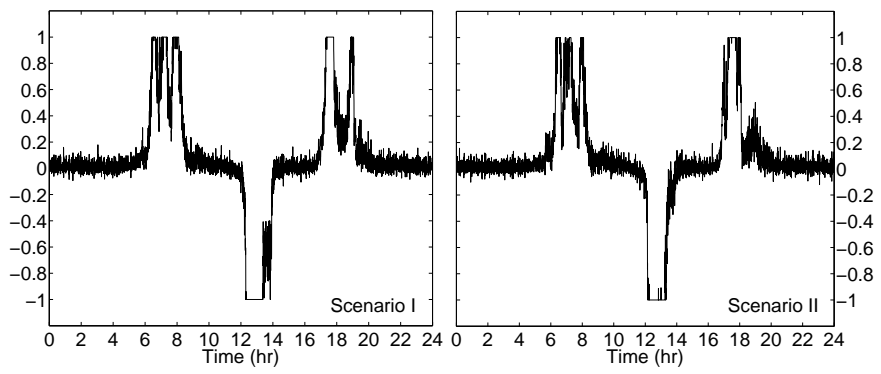

(b) $24 \mathrm{hr}$ GECN signals sent to bus 13

Fig. 9. GECN signal sent to buses 2 and 13 in the case of $20 \%$ of elastic demand with (scenario II) and without (scenario I) control of the tap-changers positions. 


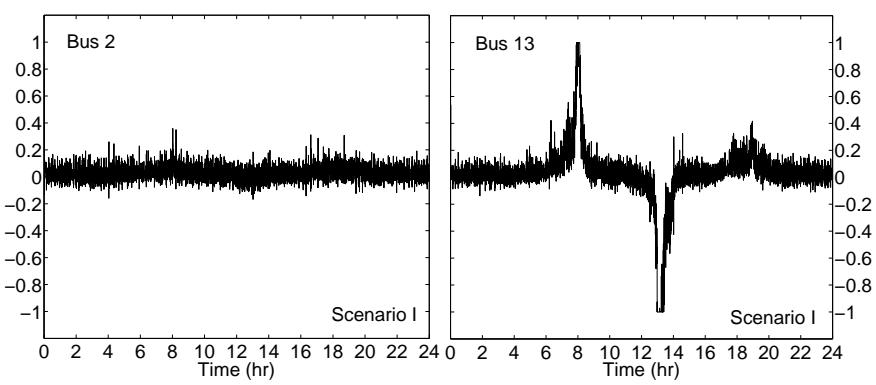

Fig. 10. GECN signal sent to buses 2 and 13 in the case of $30 \%$ of elastic demand without (scenario I) control of the tap-changers positions.

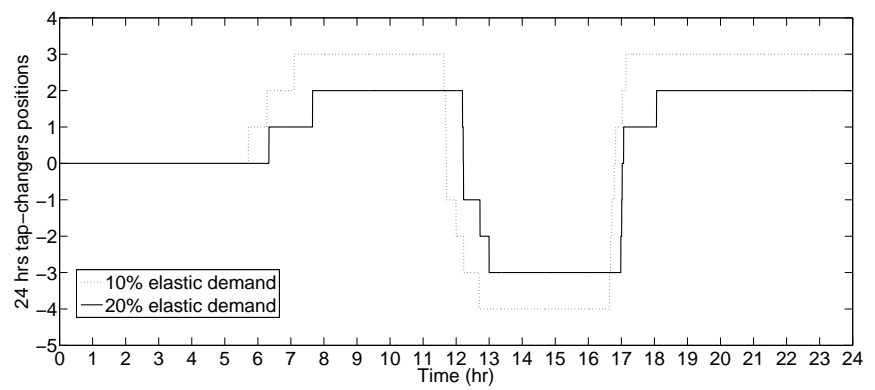

Fig. 11. 24hrs tap-changers positions for scenario II, $10 \%$ and $20 \%$ of elastic demand with control of the tap-changers.

provided by scenario II for $10 \%$ and $20 \%$ of elastic demand. For the case of $30 \%$ of elastic demand no OLTC change occurs. As expected in the case of $20 \%$ elastic demand the total number of OLTC operations is less than in the case of $10 \%$ elastic demand (12 vs 17 ). Overall, it can be observed that the obtained number of OLTC changes is compatible with a typical operation of such a device.

For the same buses (2 and 13), Fig. 12 shows the aggregate power of only the elastic appliances in correspondence of the signals presented in Fig. 8, 9 and 10 for Scenario I. This figure demonstrates that the proposed DR mechanism allows aggregated loads to follow properly the GECN signal. Also, these figures reveal that for short periods of time (in the order of 15-30 minutes) the DNO can drive the aggregate power of even a small number of elastic appliances up to twice or down to one third of their average power consumption by sending the appropriate signal.

Concerning the impact on the appliance internal states (i.e., temperature), Fig. 13 shows a histogram of the appliances' temperatures in buses 2 and 13 during different periods of the day for scenario I and $10 \%$ of elastic demand. The latter reveals that the operation of the GECN mechanism does not have an impact on the end-users quality of service, as it does not cause any deviation of the appliances temperatures outside of the allowed deadband (i.e., $3-8^{\circ} \mathrm{C}$ ). The temperatures of the elastic loads during hours $1-4$ are in compliance with the GECN signal sent that is not saturated. In the same figure, one can observe a large concentration of temperatures in the lower part of the temperature deadband during hours $11-$ 14 ; this is compatible with the signal sent during these hours



(a) Aggregate power of elastic appliances at bus 2 and 13, $10 \%$ elastic demand



(b) Aggregate power of elastic appliances at bus 2 and 13, 20\% elastic demand



(c) Aggregate power of elastic appliances at bus 2 and 13, 30\% elastic demand

Fig. 12. Aggregate power of the elastic appliances at buses 2 and 13 for scenario I, $10 \%, 20 \%$ and $30 \%$ of elastic demand and no control of the tapchangers.

to encourage consumption. The opposite effect is observed during hours $17-22$ where the peak load results in large, positive signals that dictate the reduction in consumption.

Finally, the slope of the saturation function is a significant parameter in the control law used by the DNO. In order to investigate the impact of the saturation function in the performance of the proposed DR mechanism the parameter $b$ of the saturation function is decreased to half its initial value $\left(b=1.4 E^{-3}\right)$. The signal sent to bus 3 for the two values of the parameter $b$ is presented in Fig. 14, where it is assumed that the DNO also controls the tap changers and the elastic demand in the network is $10 \%$. One can observe that, the signal does not exhibit peak values variation for the two considered cases. The sinificant impact of parameter $b$ is related to the generation of larger/smaller errors into the control loop of Fig. 2. The consequence of this is that higher/lower values of $b$ produce steeper saturation functions that result in lower/larger modifications of the GECN signal amplitude. In Fig. 15 the 


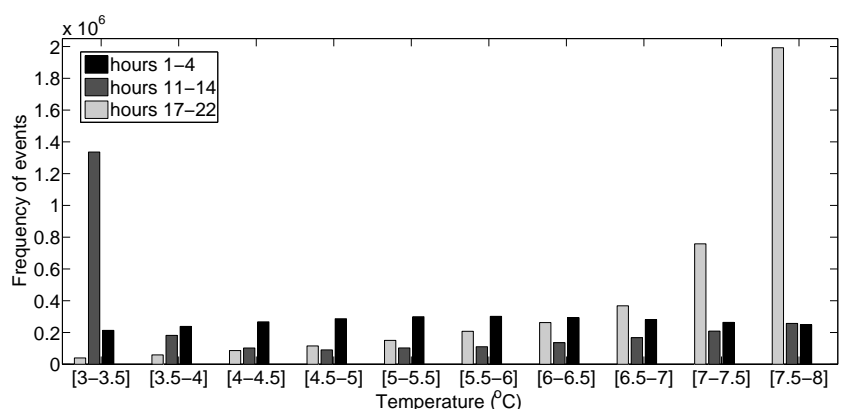

Fig. 13. Histogram of temperatures of elastic appliances (refrigerators) in buses 2 and 13 during different hours of operation for scenario I, $10 \%$ of elastic demand and no control of the tap-changers.



(a) $24 \mathrm{hr}$ signal sent to bus 3 with $b=7 E^{-4}$



(b) $24 \mathrm{hr}$ signal sent to bus 3 with $b=1.4 E^{-3}$

Fig. 14. GECN signal sent to bus 3 for different values of the parameter $b$ for scenario II, $10 \%$ of elastic demand and control of the tap-changers.

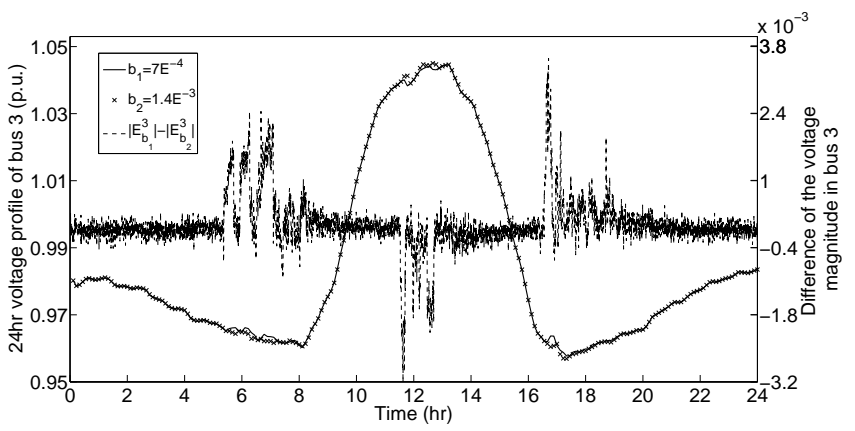

Fig. 15. Voltage profile of bus 3 for different values of the parameter $b$ for scenario II, $10 \%$ of elastic demand and control of the tap-changers.

daily voltage profile of bus 3 is shown as well as the difference in the voltage profiles for both cases of the parameter $b$. By observing Fig. 15, it is possible to conclude that the parameter $b$ does not influence the voltage quality.

\section{CONCLUSION}

This paper has proposed a new demand response mechanism for the control of thermostatic appliances (GECN). The contributions of the proposed mechanism are the following: (i) the algorithm is conceived to provide ancillary services to the grid with particular reference to primary voltage control without impacting the end users; (ii) in emergency situations such as contingencies or disturbances in the grid, the proposed scheme can be used to provide a further leverage in coordination with the DNO's own resources (e.g., OLTC).

Compared to other approaches, the one proposed in this paper assumes that the network operator does not use any other additional active source (i.e., DG or storage). Furthermore, the DNO does not have any knowledge on the parameters or the state of the elastic appliances. Instead, the resulting variation of the aggregate power at a bus level drives the control mechanism and provides the DNO with an implicit feedback that is used to estimate the responsiveness of loads.

The paper has validated the proposed DR control scheme by making reference to a typical IEEE 13 node distribution test feeder which was appropriately adapted in order to comprise a large population of heterogeneous household controllable loads along with non-elastic demand, as well as non-dispatchable power injections. The results show that the proposed GECN control mechanism is able to successfully provide primary voltage control in distribution networks. Specifically, the GECN is able to: (i) control the voltage deviations in the order of few percent of the network's voltage rated value and (ii) mitigate the use of other voltage control systems like OLTC.

\section{REFERENCES}

[1] M. Albadi and E. El-Saadany, "A summary of demand response in electricity markets," Electric Power Systems Research, vol. 78, no. 11, pp. 1989-1996, 2008

[2] J. Mathieu, M. Dyson, and D. Callaway, "Using residential electric loads for fast demand response: The potential resource and revenues, the costs, and policy recommendations," in Proceedings of the ACEEE Summer Study on Buildings, Pacific Grove, CA, Aug., 2012.

[3] T. Keep, F. Sifuentes, D. Auslander, and D. Callaway, "Using load switches to control aggregated electricity demand for load following and regulation," in Power and Energy Society General Meeting. IEEE, 2011, pp. 1-7.

[4] T. Vandoorn, B. Renders, L. Degroote, B. Meersman, and L. Vandevelde, "Active load control in islanded microgrids based on the grid voltage," IEEE Trans. Smart Grid, vol. 2, no. 1, pp. 139-151, 2011.

[5] J. Wu, J. Ekanayake, and K. Samarakoon, "Frequency response from electric vehicles," in ENERGY 2011, The First International Conference on Smart Grids, Green Communications and IT Energy-aware Technologies, 2011, pp. 148-152.

[6] K. Samarakoon, J. Ekanayake, and N. Jenkins, "Investigation of Domestic Load Control to Provide Primary Frequency Response Using Smart Meters," IEEE Trans. Smart Grid, vol. 3, no. 1, pp. 282-292, 2012.

[7] European Network of Transmission System Operators for Electricity (ENTSO-E), "Draft network code on demand connection," December $5,2012$.

[8] K. Christakou, J.-Y. LeBoudec, M. Paolone, and D.-C. Tomozei, "Efficient computation of sensitivity coefficients of node voltages and line currents in unbalanced radial electrical distribution networks," IEEE Trans. Smart Grid, vol. 4, no. 2, pp. 741-750, 2013.

[9] L. Czarnecki and Z. Staroszczyk, "On-line measurement of equivalent parameters for harmonic frequencies of a power distribution system and load," IEEE Trans. Instrumentation and Measurement,, vol. 45, no. 2, pp. 467-472, 1996. 
[10] K. Ramakrishnan, S. Floyd, and D. Black, "The Addition of Explicit Congestion Notification (ECN) to IP, September 2001. Internet Engineering Task Force (IETF)," RFC 3168, Tech. Rep.

[11] J. Taneja, D. Culler, and P. Dutta, "Towards cooperative grids: Sensor/actuator networks for renewables integration," in First IEEE International Conference on Smart Grid Communications (SmartGridComm). IEEE, 2010, pp. 531-536.

[12] S. Koch, J. Mathieu, and D. Callaway, "Modeling and control of aggregated heterogeneous thermostatically controlled loads for ancillary services," in Proc. 17th Power Syst. Comput. Conf. (PSCC2011), Stockholm, Sweden, 2011, pp. 1-7.

[13] S. Backhaus, N. Sinitsyn, S. Kundu, and I. Hiskens, "Modeling and control of thermostatically controlled loads," Los Alamos National Laboratory (LANL), Tech. Rep., 2011.

[14] N. Saker, M. Petit, and J. Coullon, "Demand side management of electrical water heaters and evaluation of the cold load pick-up characteristics (clpu)," in PowerTech, Trondheim. IEEE, 2011, pp. 1-8.

[15] N. Lu and D. Chassin, "A state-queueing model of thermostatically controlled appliances," IEEE Trans. Power Systems, vol. 19, no. 3, pp. 1666-1673, 2004.

[16] P. Constantopoulos, F. Schweppe, and R. Larson, "ESTIA: A realtime consumer control scheme for space conditioning usage under spot electricity pricing," Computers \& operations research, vol. 18, no. 8 , pp. 751-765, 1991.

[17] S. Sarri, M. Paolone, R. Cherkaoui, A. Borghetti, F. Napolitano, and C. Nucci, "State Estimation of Active Distribution Networks: Comparison Between WLS and Iterated Kalman-Filter Algorithm Integrating PMUs," in 3rd IEEE PES Innovative Smart Grid Technologies (ISGT) Europe Conference, 2012.

[18] Q. Zhou and J. Bialek, "Simplified calculation of voltage and loss sensitivity factors in distribution networks," in Proc. of the 16th Power Systems Computation Conference (PSCC2008), Glasgow, Scotland, 2008.

[19] A. Borghetti, M. Bosetti, S. Grillo, S. Massucco, C. Nucci, M. Paolone, and F. Silvestro, "Short-term scheduling and control of active distribution systems with high penetration of renewable resources," IEEE Systems Journal, vol. 4, no. 3, pp. 313-322, 2010.

[20] M. C. Ferris, "Matlab and gams: Interfacing optimization and visualization software," Mathematical Programming Technical Report, no. 98-19, p. $21,1998$.

[21] R. H. Byrd, J. Nocedal, and R. A. Waltz, "Knitro: An integrated package for nonlinear optimization," in Large-scale nonlinear optimization. Springer, 2006, pp. 35-59.

[22] M. Stadler, W. Krause, M. Sonnenschein, and U. Vogel, "Modelling and evaluation of control schemes for enhancing load shift of electricity demand for cooling devices," Environmental Modelling \& Software, vol. 24, no. 2, pp. 285-295, 2009. 